\title{
A METHOD FOR DETERMINING CARDIAC OUTPUT BY THE DIRECT FICK PRINCIPLE WITHOUT GAS ANALYSIS 1,2
}

\author{
By JACOB GROSSMAN, RAYMOND E. WESTON, AND LOUIS LEITER, WITH THE \\ ASSISTANCE OF R. S. AARON, E. R. BORUN, A. S. BUCHBERG, L. N. HALLINGER, \\ I. B. HANENSON, H. MARK, AND M. WOLFMAN
}

(From the Medical Division, Montefiore Hospital, New York, N. Y.)

(Submitted for publication September 9, 1952; accepted November 19, 1952)

With the reintroduction of cardiac catheterization (1) which facilitated the withdrawal of mixed venous blood for gas analysis, the Fick principle has been widely applied for the determination of cardiac output in man $(2,3)$. While this method provides the most accurate measurement of the cardiac output available at present and has contributed considerably to our knowledge of cardiovascular dynamics in health and disease, it is open, under certain circumstances, to several potential sources of error.

Chief among these is the requirement of gaseous equilibrium between the alveoli, blood and tissues. Any change in the alveolar gas content during the collection of exhaled air may produce not only significant error in the apparent oxygen consumption, but possibly changes in the arterial-mixed venous blood (A-MV) oxygen differences as well. Consequently, variations in respiratory function occasioned by anoxia, hyperventilation, exercise or inhalation of oxygen may lead to significant errors in the measured cardiac output. Since reestablishment of hematopulmonic equilibrium, or the steady state, following a change in respiratory function may require forty to sixty minutes (4), the immediate or early effects of altered respiratory dynamics on cardiac output cannot be evaluated.

Furthermore, the measurement of cardiac output by gas methods involves special equipment and time-consuming analyses of gases in both blood and respired air. In most laboratories, therefore, only a limited number of measurements are pos-

\footnotetext{
1 Supported in part by grants from the National Heart Institute, U. S. Public Health Service; Martha Hall Foundation, Montefiore Hospital ; Campbell Pharmaceutical Co. ; Eli Lilly \& Co.; Wyeth, Inc. ; and G. D. Searle \& Co.

2 A preliminary report was presented at the meeting of the Federation of American Societies for Experimental Biology, New York, April 17, 1952.
}

sible in a given period, and the separate determinations must be intermittent rather than continuous. Moreover, in some subjects, the periodic application of a face mask or nose clamp may produce anxiety, and thereby influence the measured cardiac output.

In order to overcome these difficulties, the possibility of using some non-gaseous test substance for measuring the cardiac output by the Fick principle was considered. The ideal substance for this purpose should possess the following characteristics: 1) rapid, fairly constant and easily measurable bodily uptake and/or excretion of sufficient magnitude to produce a significant A-MV difference; 2) ease and accuracy of determination in small quantities of body fluids by ordinary analytical methods; 3 ) known or readily verifiable volume of distribution; and 4) absence of toxicity and effects on circulatory dynamics.

When the cardiac output and the rate of removal of the test substance are constant, the magnitude of the A-MV difference is a function of two factors-the proportion of the cardiac output perfusing the extracting organ(s) and the completeness of extraction. Since the kidneys normally receive a large fraction of the cardiac output (5), any substance completely extracted by the kidneys would exhibit an adequately large A-MV difference. Moreover, renal uptake of blood constituents can be accurately measured by the urinary excretion. Sodium para-aminohippurate (PAH), which fairly well fulfills the above criteria, has a renal extraction of about 90 per cent (6). Since the normal renal fraction of the total cardiac output is about 20 per cent, the resulting anticipated A-MV difference of $\mathrm{PAH}$ would be 18 per cent $(0.20 \times 0.90)$ of the arterial concentration.

In these studies, therefore, $\mathrm{PAH}$ was first used; subsequently, to obviate difficulties arising from 
acetylation of a small fraction of the PAH (7), its acetylated derivative, para-acetylaminohippuric acid (PACA), which has the same renal clearance, was substituted. This was prepared as recommended by Newman and coworkers (8), the solutions being sterilized by passage through a Seitz filter. ${ }^{3}$

The use of a non-gaseous test substance presented a special problem. Unfortunately, the usual type of infusion into a peripheral vein cannot produce an A-MV difference, since under such circumstances, the concentration of test substance in the right side of the heart is as high or even slightly higher than in arterial blood. Only when the test substance is added to the blood of the pulmonary circulation, as is oxygen, can there result an A-MV difference. It is necessary, therefore, to introduce the test substance into the lesser circulation distal to that point from which mixed venous blood is withdrawn. Thus, if the test substance were added to the pulmonary artery and mixed venous blood removed from the right ventricle (in the absence of pulmonary regurgitation,

8 The authors are grateful to Miss J. Olch for her cooperation in filtering the preparations and testing the filtrates for sterility. interventricular septal defect, or removal in the lesser circulation), a true A-MV difference will be obtained regardless of the site of removal of the test substance. This was accomplished by placing the tip (distal lumen) of a double lumen cardiac catheter (9) into the pulmonary artery. The proximal lumen, used for the withdrawal of mixed venous blood, was located $10 \mathrm{~cm}$. back, in the outflow tract of the right ventricle just below the pulmonic valve. In Figure 1, the placement of a double lumen catheter for the determination of an A-MV difference is diagrammatically represented.

\section{DERIVATION OF FORMULAE}

The mathematical validity of the present application of the Fick principle (10) to the determination of cardiac output is established by the following derivation of the formulae used in the calculations.

The quantity of a substance circulating in a given portion of the blood flow is represented by the product of its concentration and volume of flow. In the case of a substance removed from the blood only by the kidneys, the concentration after equilibrium between blood and tissues has

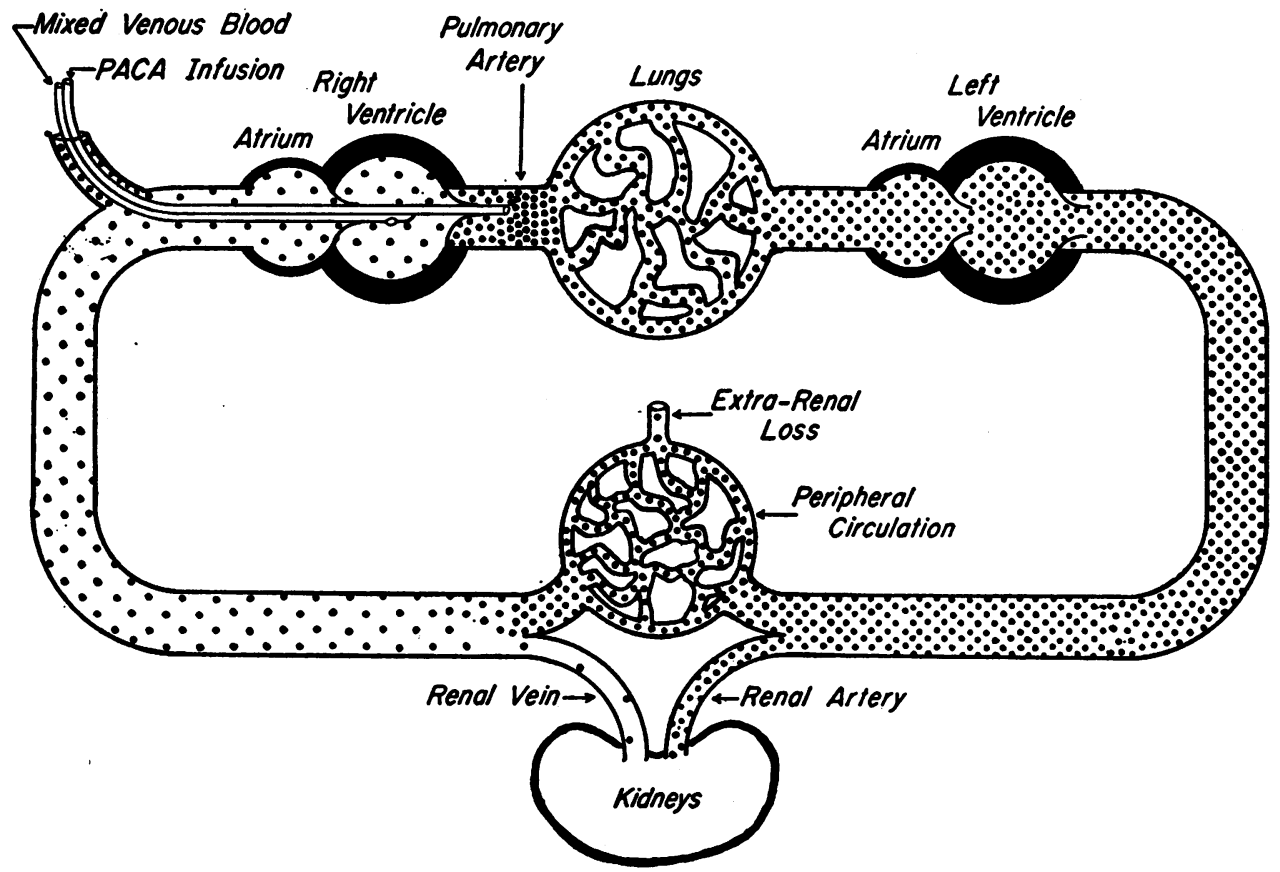

Fig. 1. Diagram Illustrating the Production of an Arterial-Mixed Venous Blood Difference by Means of a Double Lumen Cardiac Catheter 
been achieved will be the same in all vessels of the greater circulation except those veins draining the kidneys (the renal veins and inferior vena cava). Under these circumstances, the amount of substance returning to the heart per unit time, the product of mixed venous blood concentration and total body flow (cardiac output), will be the sum of that returning from the kidneys (the renal venous blood concentration times renal blood flow) plus that returning from the remainder of the body. The latter quantity may be expressed as the product of the arterial concentration and difference between the cardiac output and renal blood flow. The above may be summarized for $\mathrm{PACA}$ as in the following equation:

\section{1.}

$$
\begin{aligned}
& {[\mathrm{PACA}]_{\mathrm{MV}}(\mathrm{CO})} \\
& \text { where }=[\mathrm{PACA}]_{\mathrm{RV}}(\mathrm{RPF})+[\mathrm{PACA}]_{\Lambda}(\mathrm{CO}-\mathrm{RPF}) \text {, }
\end{aligned}
$$

$[\mathrm{PACA}]_{\mathbf{M V}, \mathrm{RV}, \mathrm{A}}$ represent the concentration of PACA in mixed venous, renal venous and arterial plasma, respectively,

$\mathrm{CO}=$ cardiac output, as plasma,

$\mathrm{RPF}=$ renal plasma flow.

Transposing, and solving for $\mathrm{CO}$,

2. $\mathrm{CO}=\frac{(\mathrm{RPF})\left([\mathrm{PACA}]_{\mathbf{A}}-[\mathrm{PACA}]_{\mathrm{RV}}\right)}{[\mathrm{PACA}]_{\mathbf{A}}-[\mathrm{PACA}]_{\mathbf{M V}}}$.

Since

3. $[\mathrm{PACA}]_{\mathrm{RV}}=(1-\mathrm{E})[\mathrm{PACA}]_{\mathrm{A}}$,

where $E=$ renal extraction of $P A C A$,

$$
\left(=\frac{[\mathrm{PACA}]_{\Lambda}-[\mathrm{PACA}]_{\mathrm{RV}}}{[\mathrm{PACA}]_{\Lambda}}\right) \text {, }
$$

Substituting in equation 2 yields

$$
\text { 4. } \begin{aligned}
\mathrm{CO} & =\frac{(\mathrm{RPF})\left([\mathrm{PACA}]_{\Lambda}-(1-\mathrm{E})[\mathrm{PACA}]_{\Lambda}\right)}{[\mathrm{PACA}]_{\Lambda}-[\mathrm{PACA}]_{\mathbf{M V}}} \\
\text { 5. } \quad & =\frac{(\mathrm{RPF})(\mathrm{E})[\mathrm{PACA}]_{\Lambda}}{[\mathrm{PACA}]_{\Lambda}-[\mathrm{PACA}]_{\mathbf{M V}}}
\end{aligned}
$$

But since

6. $(\mathrm{RPF})(\mathrm{E})=\mathrm{C}_{\mathrm{PACA}}$, the renal clearance of PACA, which $=\frac{U_{\text {PACA }}}{[\text { PACA }]_{\Lambda}}$, where UV $U_{P A O A}$ is the urinary excretion per unit time; therefore, substituting in equation 5 ,

7. $\mathrm{CO}=\frac{\frac{\mathrm{UV}_{\mathrm{PAOA}}}{[\mathrm{PACA}]_{\Lambda}} \times[\mathrm{PACA}]_{\Lambda}}{[\mathrm{PACA}]_{\Lambda}-[\mathrm{PACA}]_{\mathbf{M V}}}=\frac{\mathrm{UV}_{\mathrm{PACA}}}{[\mathrm{PACA}]_{\Lambda}-[\mathrm{PACA}]_{\mathbf{M V}}}$

which is the expression of the Fick principle for the measurement of cardiac output by the present method.

The above derivation is based upon the assumption that the removal of PACA from the plasma occurs only by renal excretion. In fact, $\mathrm{PAH}$, and presumably PACA, is excreted by way of sweat (11) and other extra-renal channels, including the liver (12). Therefore, the following modification was introduced in order to express total. plasma clearance. During the continuous infusion of a substance, at constant arterial concentration, the total amount cleared from the plasma by all routes must equal the amount administered. Under these circumstances, equation 7 may be expressed as follows:

8.

in which

$$
\mathrm{CO}=\frac{\mathrm{IV}}{[\mathrm{PACA}]_{\mathbf{\Lambda}}-[\mathrm{PACA}]_{\mathbf{u v}}}
$$

I = concentration of PACA in the infusion ( $\mathrm{mg} . / \mathrm{ml}$. ),

$\mathrm{V}=$ rate of administration of the infusion ( $\mathrm{ml} . / \mathrm{min}$.$) , and$ the product is the amount of substance infused per unit time (mg./min.).

When, because of altered cardiac output or renal hemodynamics, the steady state is disturbed and arterial concentration is not constant, a correction factor for the changing plasma level must be introduced into equation 8 . The rate of change, or slope of the line representing arterial concentration, $\mathrm{d}[\mathrm{PACA}]_{\boldsymbol{\Lambda}} / \mathrm{dt}(\mathrm{mg} . / \mathrm{ml} . / \mathrm{min}$.), is a function of the difference between the rates of plasma infusion and plasma clearance. When this is multiplied by the volume of distribution $\left(V_{D}\right)$ of the test substance, the product represents the difference between the amounts of test substance infused into and removed from the blood. Thus,

$$
\text { 9. } \mathrm{CO}=\frac{\mathrm{IV} \pm \mathrm{d}[\mathrm{PACA}]_{\Lambda} / \mathrm{dt}\left(\mathrm{V}_{\mathrm{D}}\right)}{[\mathrm{PACA}]_{\Lambda}-[\mathrm{PACA}]_{\mathbf{M V}}}
$$

during falling $(+)$ and rising $(-)$ levels, respectively.

In practice, the A-MV difference employed is one integrated throughout the desired period rather than that based upon a single pair of determinations.

The above equations have all been expressed in terms of plasma. Conversion to whole blood figures is accomplished by dividing by (1-hematocrit).

\section{MATERIAL AND METHODS}

The eighteen subjects in this series included seven without cardiac or renal disease and eleven cardiac patients, ten of whom had rheumatic heart disease with mitral stenosis.

The subjects were in the post-absorptive state, at times mildly sedated with a barbiturate. A soft rubber, multi-holed urethral catheter was inserted, and an indwelling arterial needle placed into a femoral or brachial 
artery. The double-lumen cardiac catheter was then inserted, and passed well into a branch of the pulmonary artery. A Sanborn electromanometer was connected to the proximal lumen, and the catheter slowly withdrawn during continuous recording to slightly beyond that point at which the transition from the pulmonary arterial to the right ventricular pressure tracings was observed. The proximal lumen now was located about $2 \mathrm{~cm}$. below the pulmonic valve in the outflow tract of the right ventricle. Through the distal lumen (tip), which lay about $8 \mathrm{~cm}$. into the pulmonary artery, the solution of the test substance was infused by means of a Bowman constant infusion pump at a rate of 1.0 to $3.5 \mathrm{ml}$. per minute.

Following a period of about one hour to permit equilibration of the test substance, specimens of arterial and mixed venous blood (right ventricle) and urine were obtained periodically for the continuous measurement of cardiac output by the chemical 4 method. At the same time, expired air and similar blood specimens were collected for the determination of cardiac output by the usual gas * method; the latter, however, were less frequent and intermittent. In those patients able to tolerate such activity, additional measurements were made following exercise.

Blood for oxygen determinations was collected in syringes with dead spaces obliterated by bubble-free heparin solution, and kept over mercury at $0^{\circ} \mathrm{C}$. until analyzed by the Van Slyke and Neill manometric technique (13). Duplicate determinations were required to check within 0.1 volume per cent. Expired air was collected in a Tissot spirometer, transferred to tonometers, and analyzed by the Scholander respiratory gas method (14).

PAH was determined by the method of Smith and his associates (7). PACA was measured at first by the method of Newman (8); subsequently, the acidified filtrate was hydrolyzed in $25 \mathrm{ml}$. volumetric flasks and diluted to constant volume after completing the color reaction. Hematocrits were determined on arterial blood in Wintrobe tubes centrifuged at 3,000 RPM for thirty minutes. No correction was made for trapped plasma.

\section{RESULTS}

Certain conditions and assumptions underlie the successful application of the chemical method. Firstly, competency of the pulmonic valve, particularly with the catheter in situ, should be established. Observations on the present series of patients have confirmed the reports of others that no alterations in the recorded pressure curves occur when double lumen catheters are used. In addition, to test roughly whether regurgitation occurs, Evans blue dye (T-1824) was injected into

4 The terms "chemical" and "gas," as employed in this paper, are intended simply to differentiate the two methods, since the chemical nature of each is obvious. the pulmonary artery via the distal lumen of a catheter, and blood was simultaneously withdrawn from the right ventricle. No dye was detectable in the right ventricular blood during an interval shorter than the circulation time, including that of the coronary circulation.

Secondly, success of the method requires complete mixture in the blood during passage of the test substance from the pulmonary artery to the aorta. ${ }^{5}$ Although streaming may tend to prevent complete admixture, the relatively small volume of test solution added to the large volume of blood, the complete passage through a capillary bed, and further mixing in the left chambers of the heart would favor homogeneity of the test substance throughout the arterial tree.

At first, the cardiac output was calculated from the urinary excretion of $\mathrm{PAH}$, employing equation 7 which is based upon the assumption that the urinary excretion of $\mathrm{PAH}$ reflects the total plasma clearance. However, PAH has been found in other body secretions $(11,12)$. Moreover, in our earlier studies, the cardiac output, calculated from renal excretion of either PAH or PACA, tended to be about 15 per cent less than that obtained from gas analyses. That this discrepancy reflects extrarenal plasma clearance is supported by the fact that, during constant arterial concentrations, the infusion rate exceeded the urinary excretion by about this fraction. For this reason, the cardiac outputs here reported have been calculated by the use of equation 9, or the total plasma clearance, on the basis of the rate of infusion of the test substance.

The results are summarized in Table I and Figure 2. In general, the renal plasma flows and cardiac outputs conform with those previously reported (15). Among the rheumatic patients with mitral stenosis, both the circulatory and the renal hemodynamics, as well as the respiratory data, are in good agreement with those of Werkö and his colleagues $(16,17)$. Thus, the clearances of $\mathrm{PAH}$ and PACA in the cardiac patients are about one-third of those of the non-cardiac subjects. Moreover, the decrease in renal blood flow and in

5 The same assumption is inherent in the dye or isotope dilution methods, especially since rapid injection is made. Actually, the lower molecular weight of PACA and slow rate of injection may be expected to result in more rapid mixing. 
renal fraction of the cardiac output bears a rough relationship to the clinical estimate of the severity of failure. The oxygen consumption at rest is equivalent in both groups, but the oxygen uptake per liter of air ventilated is lower in the cardiac patients, a reflection of the respiratory dysfunction.

The cardiac outputs for corresponding collection periods indicate good agreement between the two methods. (Correlation coefficient, $r=0.90$.) After use of the constant infusion pump resulted in more constant arterial levels, even better agreement was attained.

In one patient, G. M., a 26 year old male with sarcoidosis and pulmonary fibrosis, the effect of changing the gas content of the respired air on the determination of cardiac output by the gas and chemical methods was studied. Following control measurements while breathing from a tank of compressed air, a moderate degree of hypoxia was produced by permitting the subject to inhale a mixture of 10 per cent oxygen and 90 per cent nitrogen for 51 minutes. During this interval, the cardiac output was measured twice by the gas method and continuously by the chemical method (three urinary collection periods). Following return to the breathing of the air from the tank, additional measurements were made. The results are indicated in Figure 3.
Although there was generally good agreement between the two methods, certain differences were observed. During the hypoxic period, the output, as determined by the chemical method, rose initially to a maximal value of $12.1 \mathrm{1} . / \mathrm{min}$. then fell gradually to its previous level. At the same time, the cardiac output by the gas method rose more slowly so that the higher value, $11.051 . / \mathrm{min}$., was obtained toward the end of the hypoxic period. Following thirty minutes of inhalation of ambient air, the cardiac outputs, as measured by both methods, were at control levels.

The substitution of PACA for PAH was based upon previous reports $(7,8)$ that a small, but variable, fraction of the PAH is acetylated in the body. Such acetylation, contributing to extra-renal loss, would produce an error in the cardiac output as calculated from urinary excretion. In several studies, PAH was infused and the plasma and urinary concentrations of both PAH and PACA were determined by analyzing the plasma filtrates and diluted urine specimens both prior to and following hydrolysis. Although no significant amounts of PACA were present in arterial blood, the urine of these patients contained substantial amounts of the acetylated compound, sufficient, in fact, to make it appear that renal acetylation of

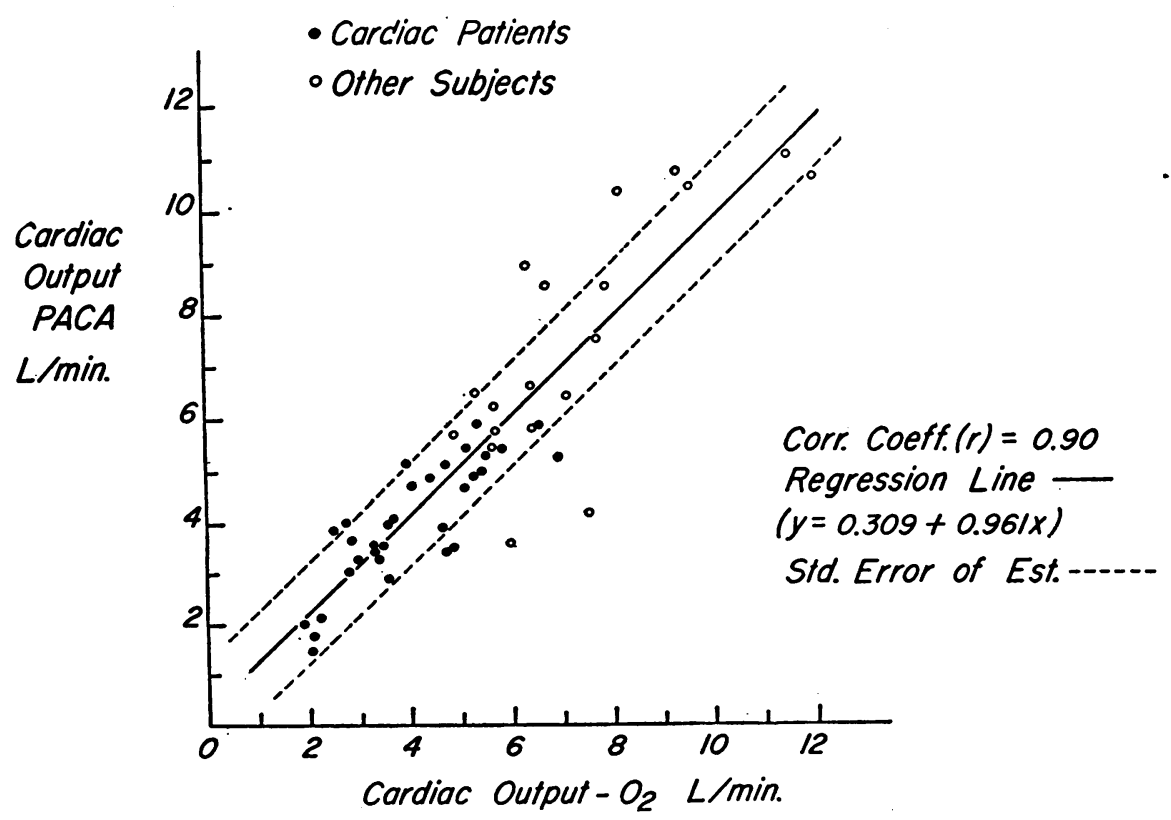

Fig. 2. Correlation Between the Cardiac Outputs as Determined by the Gas (OXYGen) and Chemical (PACA) Methods 
TABLE I

Summary of data

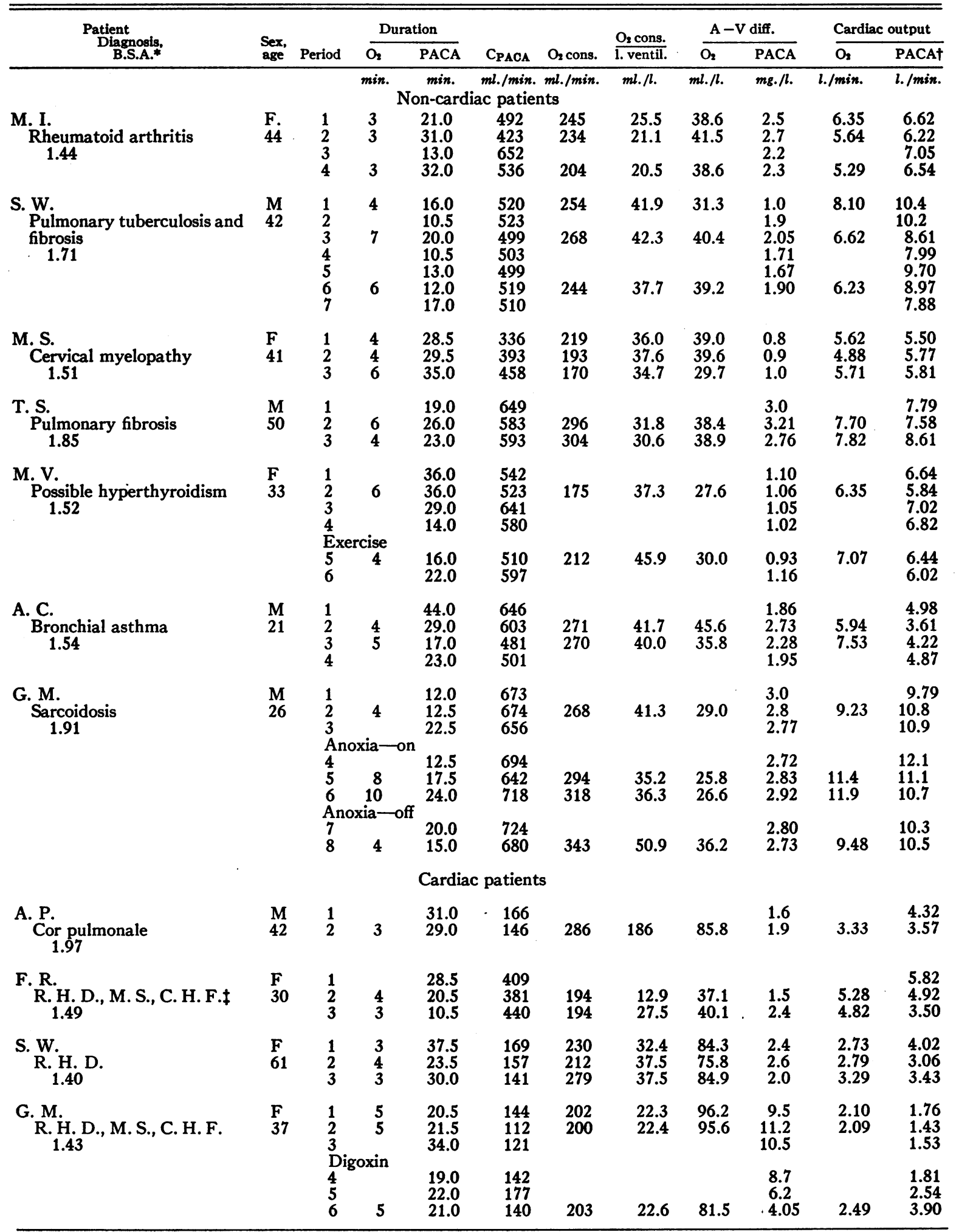


TABLE I-Continued

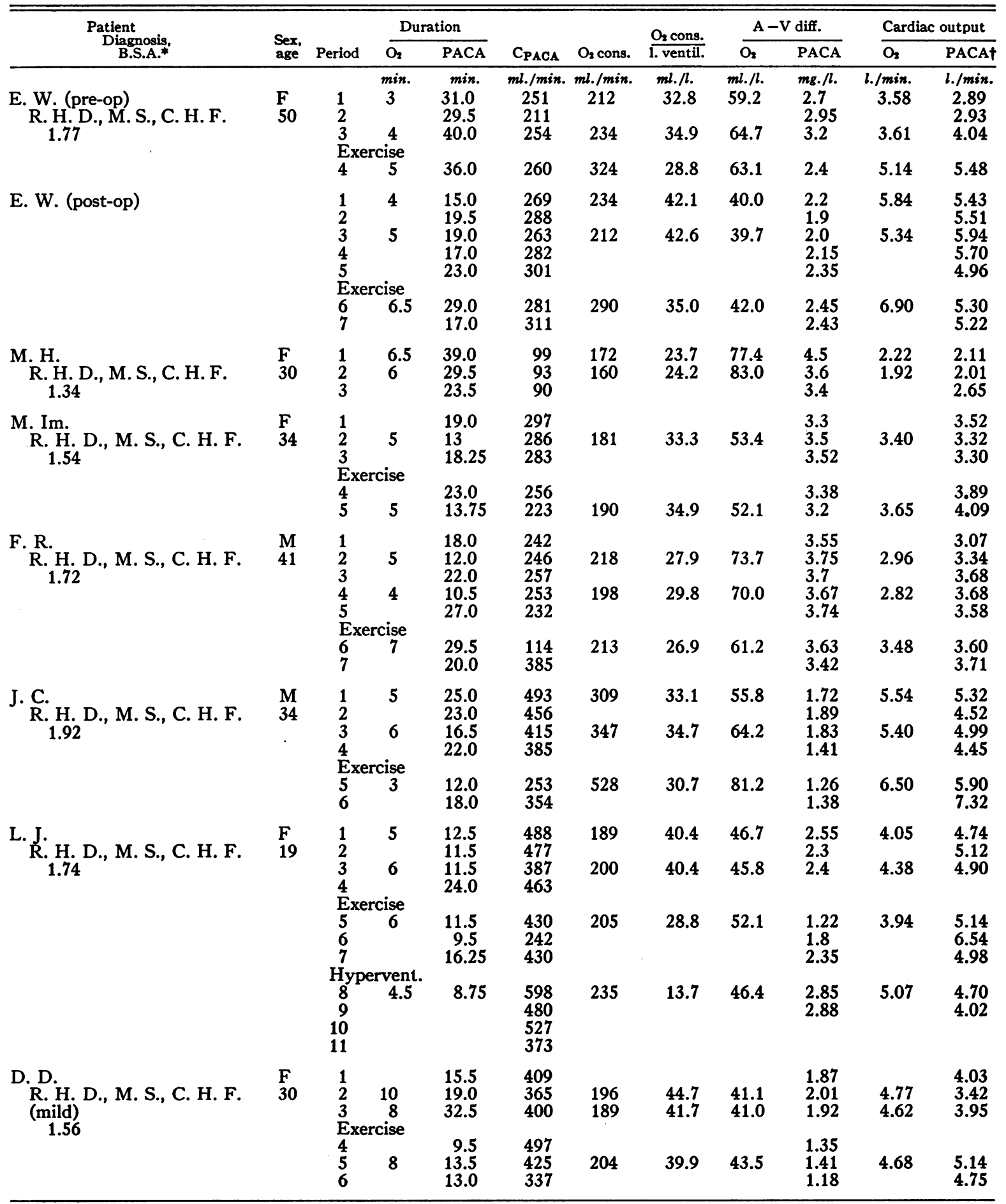

* B.S.A. = Body surface area $\left(\mathrm{M}^{2}\right)$.

t Based upon the total plasma clearance as calculated from the infusion rate and arterial plasma concentration of PACA (equation 9) and the integrated A-MV difference.

$\ddagger$ R.H.D. - Rheumatic heart disease.

M.S.-Mitral stenosis.

C.H.F.-Congestive heart failure. 
G.M. O०

Sarcoidosis

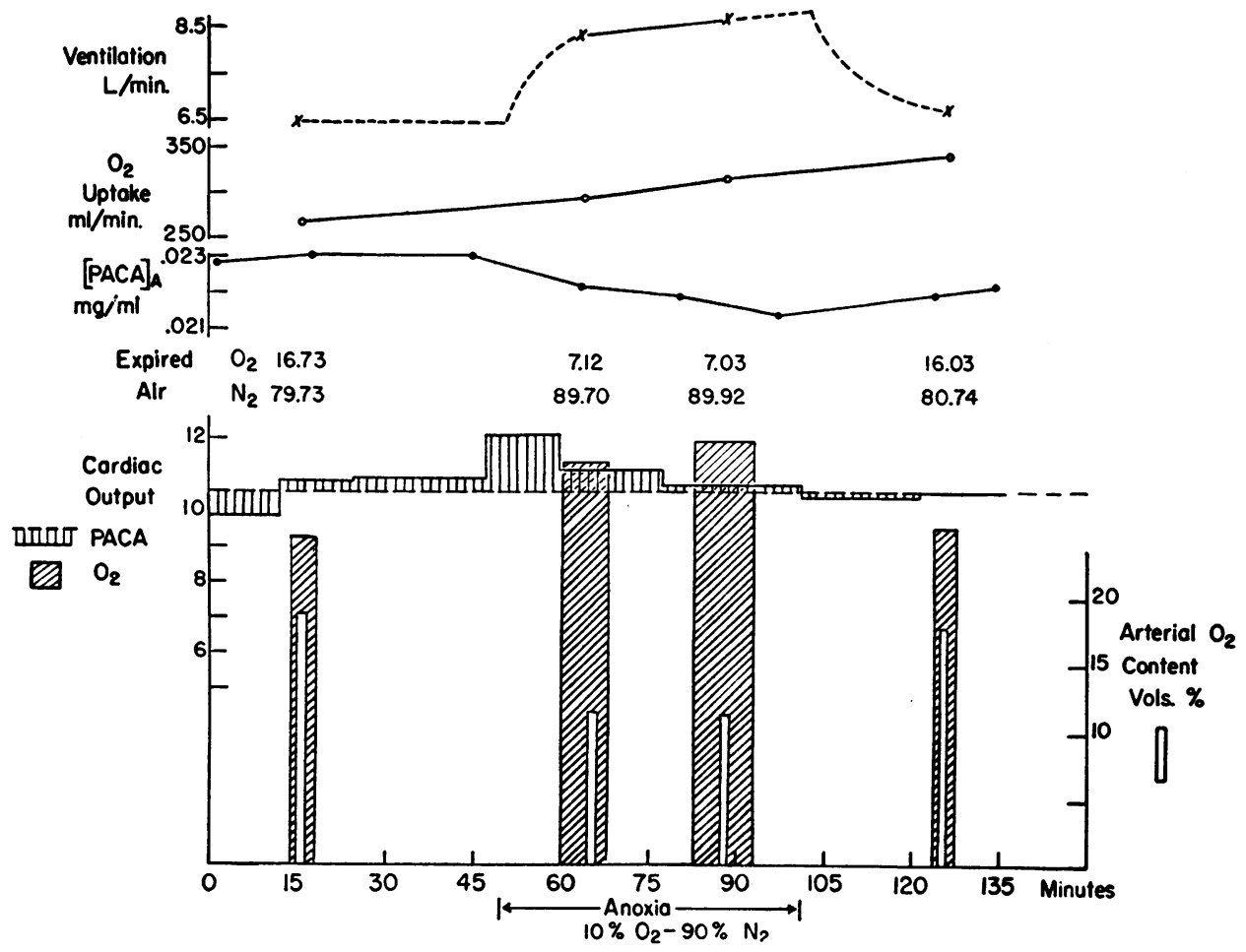

Fig. 3. Efrect of Hypoxemia on the Cardiac Output as Measured by the Gas and Chemical Methods

PAH must occur. These data may be included in a separate report.

\section{DISCUSSION}

The evaluation of accuracy of any new method for the measurement of a physiological function usually requires comparison with some previously employed technique or method which, itself, should but may not be accurate. For example, the earlier methods of cardiac output determination involved such large error that the later, more refined measurements differed significantly (18). Despite this fact, they gained general acceptance.

Because both the chemical and gas methods utilize the Fick principle, based upon the actual withdrawal of mixed venous blood, generally good agreement between the two measurements of cardiac output, as observed in the present series, might have been anticipated. However, several factors may lead to certain differences in the re- sults. Firstly, since the measured flow represents an average for the time of the determination, slight differences in the calculated cardiac output may result when the periods of simultaneous measurement by the two methods are not of the same duration. In order to compare the results based upon urinary excretion (equation 7) with those from the rate of infusion (equation 9), the cardiac outputs by the PACA method were arbitrarily calculated for the ten to forty minute periods of urine collection. The fact that the other cardiac outputs were based upon the usual three to six minute gas collection periods might have contributed to the observed differences. It should be emphasized, however, that outputs based upon the rate of infusion and integrated A-MV differences of the test substance require no urine collections, and may be calculated for considerably shorter periods.

Secondly, the apprehension and anxiety produced by the nose clamp and mouth piece tended to increase the cardiac output during the period of 
expired gas collection above that obtained during the longer interval of urine collection.

Thirdly, since the hematocrit is employed in the calculations of the chemical method, correction for trapped plasma would have lowered the measured output by 1 to 2 per cent.

The good agreement between the results of the two methods notwithstanding the above factors supports the validity of the chemical dilution method.

For the successful application of the Fick principle, maintenance of a state of equilibrium is required if the $\mathrm{A}-\mathrm{V}$ difference is to reflect accurately the change in blood flow. Because the A-MV difference is usually small, slight absolute variations in the concentration of either the arterial or mixed venous blood, due either to analytical error or to changing levels, may produce large errors in the calculated flow.

In the gas method, any variation of cardiac or respiratory function, or the content of the respired gases, will be reflected both in the measured gas utilized or produced and the A-MV difference, until a steady state, or equilibrium between the blood gas and alveolar air, has been reestablished (19). For example, with a sudden change from breathing ambient air to a 10 per cent oxygen-90 per cent nitrogen mixture, the spurious high oxygen content of the exhaled gas resulting from exhalation of oxygen previously inhaled, will produce a falsely low figure for oxygen utilization. Simultaneously, as alveolar oxygen concentration decreases, the rate of fall in oxygen concentration of arterial as compared with that of mixed venous blood also will affect the A-MV difference. To the extent that these rates differ, a spurious difference will be obtained.

Similarly, a change from room air to 100 per cent oxygen inhalation will also affect the measurement of A-MV differences and oxygen consumption. Moreover, hyperventilation, tachypnea of exercise, and other respiratory or cardiac fluctuations affecting pulmonary gas content will produce errors of varying degree in cardiac output determination until near equilibrium is attained.

By the same token, sudden changes in the cardiac output, renal blood flow or renal fraction will lead to changes in the arterial and mixed venous blood concentrations of PACA administered at a constant rate until re-equilibration occurs. Thus, during rising blood levels, the movement of PACA from plasma into interstitial fluid will produce an increased A-MV difference and, therefore, falsely low output; conversely, during falling levels, the diffusion of PACA from the extracellular fluid into the blood will yield a low A-MV difference and apparently high output. It is evident, therefore, that in the chemical adaptation of the Fick principle, as well as in the gas method, the absence of equilibrium or steady state may be a source of significant error.

However, except in the presence of markedly changing renal blood flow, the slopes of the arterial and mixed venous plasma levels are small, and the effects of PACA redistribution likewise relatively less. In such cases, more frequent withdrawal of blood specimens, the use of longer periods for measurement of cardiac output, and the application of the slope correction factor further reduce possible error. ${ }^{\circ}$ Moreover, the low molecular weight and rapid diffusibility of PACA and the extensive capillary and cell membrane surface area for transfer promote rapid equilibration between blood, extracellular fluid and tissues except in very rapid changes of blood concentration.

Thus, the present method, although subject to the same errors resulting from dysequilibria, unlike the gas method, is less influenced by changes in respiratory function. Consequently, it may be of special value in recording early changes in cardiac output during respiratory changes without the delay required for pulmonary equilibration, provided that renal flows are not significantly altered. Conversely, the gas method would be preferable for studying the effect on cardiac output of drugs like epinephrine which produce a marked change in renal blood flow, thus upsetting the PACA equilibrium.

The observations on the effects of hypoxia in patient G. M. illustrate these points. Whereas the chemical method indicated an immediate rise in cardiac output with the induction of hypoxia, followed by a return to the control level, the gas

- This problem may be further complicated, in the case of substances like PACA, which enter certain cells. When this migration is very rapid, it has little effect on the $A-V$ difference. Should the equilibration time across cell membranes be prolonged relative to that across the capillary membranes, longer periods must be employed to obtain true A-MV differences and accurate outputs. 
method indicated a gradual rise of cardiac output throughout the period of hypoxia, falling only after return to breathing air. The reasons for the lower gas output on shifting from higher to lower oxygen breathing have been discussed above. It is of interest that earlier studies from Cournand's laboratory (20) had indicated decreased cardiac output with hypoxia. This result was re-interpreted later (19), when the implications of the steady state were better understood. In the present study, permitting more time for equilibration during the hypoxic period prior to measurement of cardiac output would have resulted in failure to demonstrate any significant change. Thus, Lewis and Gorlin (21) produced an equivalent degree of hypoxia in dogs, and after allowing time for equilibration, found no change in cardiac output.

The relatively simple operations involved in the chemical method provide a certain elasticity not present in the gas method, which ordinarily requires mask, Tissot spirometer or Douglas bag, air tank, tonometers, etc. In contrast, with the catheter in situ and the constant infusion running, the chemical method requires merely periodic withdrawal of arterial and mixed venous blood, since even urine collections are not necessary. This procedure is painless and can easily be accomplished under diverse conditions, although it does not obviate the need for cardiac catheterization.

The critical influence of the A-MV difference on the accuracy of any measurement by the Fick principle should be re-emphasized. Of special importance are the variations in A-MV difference with the level of cardiac output. In congestive failure, as the cardiac output falls, with either unchanged or somewhat increased oxygen consumption, the A-MV difference rises considerably. Consequently, the accuracy of the gas method is actually increased in cardiac subjects. Under similar circumstances, the chemical method employing PACA behaves quite differently. Because of the decreased renal plasma flow, excretion of PACA decreases so that any tendency for the A-MV difference to rise with falling output is counteracted. In fact, the greatly diminished renal fraction of the total blood flow reduces the A-MV difference. The PACA method, therefore, is least accurate in conditions with low cardiac output and decreased renal fraction. Conversely, where the cardiac output is high without change in oxygen consumption or renal fraction, the gas method is less and the chemical method relatively more accurate. Therefore, the present studies on a group of subjects predominantly with advanced mitral disease and congestive failure subjected the chemical method to a most severe test. That correlation remained good even under these conditions attests to the validity of the method.

A more quantitative evaluation of the two methods in terms of the A-MV difference may be made by comparing the ratios of the A-MV difference of each to the error of determination of the blood concentration. ${ }^{7}$ Assuming a 1 per cent error in the PACA method, the quotient in normal subjects will be 15 to 18 . (i.e., 15 to $18 \% / 1 \%$ ) ; similarly, a 0.1 volume per cent error in blood gas analyses will yield a value of about 40 (4 vols. \%) 0.1 vol.\%) and a 0.2 volume per cent error, of 20 . Thus, under normal conditions, the accuracy of the gas method would be expected to be greater than that of the chemical method employing PACA.

However, further improvement of the chemical method may yet be attained. For example, the use of a different test substance with large general tissue uptake not limited to the kidneys would result in much higher A-MV differences. It is essential that the rate of uptake be fairly constant, the analysis simple and accurate, and equilibrium rapidly attained. Several substances including fructose and some isotopically labelled compounds are being considered.

In a sense, then, the results herein reported, while supporting the inherent validity of the chemical method, are preliminary. Further refinement and greater accuracy would appear to be readily capable of achievement.

\section{SUMMARY}

1. A method in which the direct Fick principle for the measurement of cardiac output is modified for the use of a non-gaseous test substance is described.

7 The fraction, A-MV difference/analytic error, is simply an index of the accuracy of the method. In practice, in each method, since two analyses determine the A-MV difference, the actual error is doubled. The fraction employed is simply for comparison. 
2. In a series of 18 patients, 11 of whom had heart disease with congestive failure of varying severity, this method was employed together with the usual oxygen method and good correlation $(r=0.90)$ was obtained. In one patient, the effect of moderate hypoxemia on the determination by both methods was studied.

3. The relative merits of the two methods under conditions of respiratory dysequilibria, varying cardiac output and renal fraction are discussed.

\section{ACKNOWLEDGMENT}

The authors wish to thank Mr. George Ross and Mr. Antol Herskovitz for aid in the preparation of the figures.

\section{REFERENCES}

1. Cournand, A., and Ranges, H. A., Catheterization of the right auricle in man. Proc. Soc. Exper. Biol. \& Med., 1941, 46, 462.

2. Cournand, A., Riley, R. L., Breed, E. S., Baldwin, E. de F., and Richards, D. W., Jr., Measurement of cardiac output in man using the technique of catheterization of the right auricle or ventricle. J. Clin. Invest., 1945, 24, 106.

3. Stead, E. A., Jr., Warren, J. V., Merrill, A. J., and Brannon, E. S., The cardiac output in male subjects as measured by the technique of right atrial catheterization. Normal values with observations on the effect of anxiety and tilting. J. Clin. Invest., 1945, 24, 326.

4. Rahn, H., and Otis, A. B., Alveolar air during simulated flights to high altitudes. Am. J. Physiol., 1947, 150, 202.

5. Smith, H. W., The Kidney. Structure and Function in Health and Disease. Oxford University Press, New York, 1951, p. 551-2.

6. Ibid., Table III, p. 161.

7. Smith, H. W., Finkelstein, N., Aliminosa, L., Crawford, B., and Graber, M., The renal clearances of substituted hippuric acid derivatives and other aromatic acids in dog and man. J. Clin. Invest., 1945, 24, 388.

8. Newman, E., Kattus, A., Genecin, A., Genest, J., Calkins, E., and Murphy, J., Observations on the clearance method of determining renal plasma flow with diodrast, para-aminohippuric acid (PAH), and para-acetyl-aminohippuric acid (PACA). Bull. Johns Hopkins Hosp., 1949, 84, 135.

9. Cournand, A., Bloomfield, R. A., and Lauson, H. D., Double lumen catheter for intravenous and intracardiac blood sampling and pressure recording. Proc. Soc. Exper. Biol. \& Med., 1945, 60, 73.

10. Fick, A., Uber die messung des blutquantums in den herzventrikeln. Verhandl. d. Phys.-Med. Ges. zu Würzburg, N. F., 1870, 2, XVI. Cited by Hoff, H. E., and Scott, H. J., Physiology (continued). New England J. Med., 1948, 239, 122.

11. Brandt, J. L., Personal communication.

12. This laboratory, Unpublished observations.

13. Van Slyke, D. D., and Neill, J. M., The determination of gases in blood and other solutions by vacuum extraction and manometric measurement. I. J. Biol. Chem., 1924, 61, 523.

14. Scholander, P. F., Analyzer for accurate estimation of respiratory gases in one-half cubic centimeter samples. J. Biol. Chem., 1947, 167, 235.

15. Merrill, A. J., Edema and decreased renal blood flow in patients with chronic congestive heart failure: evidence of "forward failure" as the primary cause of edema. J. Clin. Invest., 1946, 25, 389.

16. Werkö, L., Ek, J., Bucht, H., and Eliasch, H., Correlation between renal dynamics, cardiac output and right heart pressures in mitral valvular disease. Scandinav. J. Clin. \& Lab. Invest., 1952, 4, 15.

17. Eliasch, H., The pulmonary circulation at rest and on effort in mitral stenosis. Scandinav. J. Clin. \& Lab. Invest., 1952, 4, Suppl. 4.

18. Grollman, A., The Cardiac Output of Man in Health and Disease. Charles C. Thomas, Springfield, Ill., 1932.

19. Cournand, A., Some aspects of the pulmonary circulation in normal man and in chronic cardiopulmonary diseases. Circulation, 1950, 2, 641.

20. Motley, H. L., Cournand, A., Werko, L., Himmelstein, A., and Dresdale, D., The influence of short periods of induced acute anoxia upon pulmonary artery pressures in man. Am. J. Physiol., 1947, 150, 315.

21. Lewis, B. M., and Gorlin, R., Effects of graded hypoxemia on the pulmonary circulation of the dog. II. Moderate hypoxemia. Federation Proc., 1952, $11,93$. 